\title{
VERIFICAC̣ÃO DAS DOSES DE RADIAC̣ÃO ABSORVIDAS DURANTE A TÉCNICA DE IRRADIAÇÃO DE CORPO INTEIRO NOS TRANSPLANTES DE MEDULA ÓSSEA, POR MEIO DE DOSÍMETROS TERMOLUMINESCENTES*
}

\author{
Adelmo José Giordani ${ }^{1}$, Helena Regina Comodo Segreto ${ }^{2}$, Roberto Araújo Segreto ${ }^{3}$, \\ Regina Bitelli Medeiros ${ }^{4}$, José Salvador R. de Oliveira $^{5}$
}

Resumo OBJETIVO: Avaliar a precisão das doses de radiação absorvidas na terapia de transplantes de medula óssea durante a técnica de irradiação de corpo inteiro. MATERIAIS E MÉTODOS: Utilizaram-se 200 pastilhas de sulfato de cálcio com disprósio compactado com teflon ( $\mathrm{CaSO}_{4}+$ teflon), calibradas no ar e no "phantom", selecionadas aleatoriamente e dispostas em grupos de cinco no corpo dos pacientes. As leituras dosimétricas foram efetuadas pela leitora Harshaw 4000A. Nove pacientes foram irradiados no corpo inteiro em paralelos e em opostos laterais, utilizando-se unidade de cobalto-60, modelo Alcion II, com taxa de dose de $0,80 \mathrm{~Gy} /$ $\min$ a $80,5 \mathrm{~cm},\left\{\mathrm{campo}(10 \times 10) \mathrm{cm}^{2}\right\}$. A dosimetria dessa unidade foi realizada com dosímetro Victoreen 500. Para a determinação da dose média em cada ponto avaliado usaram-se os fatores individuais de calibração das pastilhas no ar e no "phantom", colocando-se um "build up" de $2 \mathrm{~mm}$ para superficializar a dose à distância de $300 \mathrm{~cm}$. RESULTADOS: Em 70\% dos pacientes obteve-se variação de dose menor que $5 \%$ e em $30 \%$ dos pacientes essa variação foi inferior a $10 \%$, quando comparados os valores medidos com aqueles calculados em cada ponto. Na cabeça ocorre absorção, em média, de $14 \%$ da dose administrada, e nos pulmões, acréscimo de $2 \%$ na dose administrada. Nos pacientes com distância látero-lateral maior que 35 $\mathrm{cm}$ as variações entre as doses calculadas e medidas podem chegar a $30 \%$ da dose desejada, sem o uso de filtros compensadores. CONCLUSÃO: Os valores medidos das doses absorvidas nos diversos pontos anatômicos, comparados aos valores desejados (teóricos), apresentam tolerância de $\pm 10 \%$, considerando-se as diferenças anatômicas existentes, quando utilizados os fatores de calibração individuais das pastilhas. Unitermos: Irradiação de corpo inteiro; Dosimetria; Dosímetro termoluminescente; Doses; Transplante de medula óssea.

\begin{abstract}
Measurement of absorbed radiation doses during whole body irradiation for bone marrow transplants using thermoluminescent dosimeters.

OBJECTIVE: To evaluate the precision of the absorbed radiation doses in bone marrow transplant therapy during whole body irradiation. MATERIALS AND METHODS: Two-hundred $\mathrm{CaSO}_{4}$ :Dy + teflon tablets were calibrated in air and in "phantom". These tablets were randomly selected and divided in groups of five in the patients' body. The dosimetric readings were obtained using a Harshaw 4000A reader. Nine patients had their entire bodies irradiated in parallel and opposite laterals in a cobalt-60 Alcion II model, with a dose rate of $0.80 \mathrm{~Gy} / \mathrm{min}$ at $80.5 \mathrm{~cm},\left\{(10 \times 10) \mathrm{cm}^{2}\right.$ field $\}$. The dosimetry of this unit was performed using a Victoreen 500 dosimeter. For the determination of the mean dose at each point evaluated, the individual values of the tablets calibrated in air or "phantom" were used, resulting in a build up of $2 \mathrm{~mm}$ to superficialize the dose at a distance of $300 \mathrm{~cm}$. RESULTS: In 70\% of the patients a variation of less than $5 \%$ in the dose was obtained. In $30 \%$ of the patients this variation was less than $10 \%$, when values obtained were compared to the values calculated at each point. A mean absorption of $14 \%$ was seen in the head, and an increase of $2 \%$ of the administered dose was seen in the lungs. In patients with latero-lateral distance greater than 35 $\mathrm{cm}$ the variation between the calculated doses and the measured doses reached $30 \%$ of the desired dose, without the use of compensation filters. CONCLUSION: The measured values of the absorbed doses at the various anatomic points compared to the desired doses (theoretic) presented a tolerance of $\pm 10 \%$, considering the existent anatomical differences and when using the individual calibration factors of the tablets. Key words: Whole body irradiation; Dosimetry; Thermoluminescent dosimeters; Doses; Bone marrow transplantation.
\end{abstract}

* Trabalho realizado no Setor de Radioterapia da Universidade Federal de São Paulo/Escola Paulista de Medicina (Unifesp/EPM), São Paulo, SP.

1. Físico Responsável pela Radioproteção e Física Médica do Setor de Radioterapia da Unifesp/EPM.

2. Professora Adjunta do Departamento de Medicina, Coordenadora do Laboratório de Radioterapia Experimental "Prof. Dr. Camillo Segreto" do Setor de Radioterapia da Unifesp/EPM.
3. Professor Adjunto Livre-Docente do Departamento de Medicina, Chefe do Setor de Radioterapia da Unifesp/EPM.

4. Professora Adjunta, Chefe da Coordenadoria de Física e Higiene das Radiações do Departamento de Diagnóstico por Imagem da Unifesp/EPM.

5. Professor Adjunto, Chefe do Transplante de Medula Óssea do Departamento de Medicina da Unifesp/EPM.

Endereço para correspondência: Adelmo José Giordani. Uni- versidade Federal de São Paulo/Escola Paulista de Medicina Radioterapia. Rua Napoleão de Barros, 715, subsolo, Vila Clementino. São Paulo, SP, 04024-002.E-mail: adelmogiordani@ ig.com.br.

Recebido para publicação em 11/8/2003. Aceito, após revisão, em 23/10/2003. 


\section{INTRODUÇÃO}

No final do século 19, fatos relevantes para a história das radiações ionizantes ocorreram: a descoberta dos raios X (RX) por Wilhelm Conrad Röntgen, em 1895, a constatação da radioatividade natural presente no urânio por Henry Becquerel, em 1896, seguida da separação do polônio e rádio, em 1898, pelo casal Curie ${ }^{(\mathbf{1})}$. Nessa época de grande empirismo, pesquisadores franceses, ingleses e alemães, usando exposição aleatória de corpo inteiro a radioisótopos ou ampolas de RX, introduziram as bases da atual radiobiologia clínica ${ }^{(2)}$.

As técnicas de irradiação de campos amplos ou alagardos foram introduzidas em 1923, para tratamento de diferentes doenças $^{(3)}$. Em 1927, Teschendorf relatou resultados favoráveis ao tratamento de doenças malignas, especialmente linfomas, método conhecido como "Teschendorf method"(4). Heublein, em 1932, utilizou máquina de RX de 185 kVp, 3 mA, com filtro de $2 \mathrm{~mm}$ de cobre, para tratar pacientes com dose próxima a $750 \mathrm{R}$, equivalente à "dose eritema", método que foi denominado "Heublein therapy"(4,5).

Em relação à técnica da irradiação de corpo inteiro (ICT), também conhecida como forma de tratamento sistêmico, esta remonta do início do século passado, quando não se contava com drogas quimioterápicas efetivas $^{(2)}$. O fato de uma dose única de radioterapia da ordem de $3 \mathrm{~Gy}^{(\mathbf{2 , 4})}$ aplicada em todo corpo ser potencialmente letal e determinar severa depressão medular fez com que esse tipo de tratamento fosse restrito a pacientes terminais como método paliativo. O pequeno sucesso desta modalidade de tratamento, juntamente com o desenvolvimento de drogas quimioterápicas ativas no final da década de 50, diminuíram ainda mais as indicações da técnica de $\mathrm{ICT}^{(\mathbf{2 , 3})}$. Posteriormente, modificações nos protocolos de ICT, com o uso de doses fracionadas de radiação, foram introduzidas ${ }^{(6,7)}$. Estas, aliadas ao desenvolvimento de antibióticos potentes e fatores de crescimento da medula óssea, permitiram o uso desta técnica de irradiação com relativo sucesso ${ }^{(\mathbf{8 , 9})}$.

Atualmente, uma das principais indicações da ICT é para o condicionamento do transplante de medula óssea (TMO). Estes podem ser singênicos, quando efetuados entre irmãos gêmeos univitelinos, e alogênicos, quando entre irmãos não-gêmeos mas idênticos quanto aos antígenos do complexo maior de histocompatibilidade sistema HLA. Pode também ser realizado o transplante autólogo, quando a medula do próprio paciente é colhida, tratada ou não in vitro, conforme a doença de base, criopreservada e infundida no paciente. Células hemocitopoéticas jovens também podem ser infundidas por administração de sangue do cordão umbilical ${ }^{(2)}$.

A finalidade da ICT é realizar a imunossupressão dos pacientes, promover a citorredução e criar espaço para o desenvolvimento da nova medula transplantada ${ }^{(\mathbf{1 0})}$. A importância de cada um desses efeitos depende da doença e do tipo de transplante que será realizado. Genericamente, a função imunossupressora da ICT é bastante importante nos transplantes alogênicos, pois é necessário diminuir a rejeição do enxerto, e pouco relevante quando esse não for o problema primordial, como nos transplantes autólogos ${ }^{(6,7)}$. A ICT faz parte da maioria dos protocolos de condicionamento para os TMO alogênicos ${ }^{(6-11)}$. A função de destruir as células doentes ou promover a citorredução depende da carga tumoral inicial e do tratamento combinado de quimioterapia e radioterapia usado para o condicionamento do paciente ${ }^{(\mathbf{8 , 9})}$. No caso de pacientes portadores de tumores sólidos avançados, em que a carga tumoral é bastante elevada, muitas vezes é indicada a ICT associada à quimioterapia e posterior $\mathrm{TMO}^{\mathbf{( 1 2 )}}$. Nesse contexto, a citorredução pela ICT é um objetivo importante.

A vantagem da ICT nas doses preconizadas é que o procedimento diminui bastante a rejeição, sem abolir completamente o processo ${ }^{(\mathbf{1 2})}$. Na verdade, a doença do enxerto versus hospedeiro, em graus leves, propicia também uma resposta desejada no processo de cura, favorecendo, por exemplo, a destruição de célula leucêmica residual $^{(13,14)}$.

A dose de radiação necessária para que seja alcançada a eficácia do tratamento em procedimentos com resgate com medula óssea ou com sangue periférico de parentes totalmente HLA compatíveis varia entre 8 e $10 \mathrm{~Gy}$, quando se usa fração única, com baixa taxa de dose (inferior a 5 Gy/ $\min )^{(\mathbf{1 5})}$. Nos esquemas hiperfracionados, a dose de 2 Gy é utilizada em duas sessões ao dia, com dose total de 12 Gy, ou 1,2 Gy em três sessões ao dia, com dose total de 13,2 $\mathrm{Gy}^{(\mathbf{8 , 1 6})}$. Nos casos em que se utiliza o resgate com medula óssea ou com sangue não totalmente compatíveis por meio do sistema HLA, a dose necessária parece ser superior a $12 \mathrm{~Gy}^{(\mathbf{8 , 1 7})}$. Em qualquer das seleções mencionadas, a dose é superior à dose letal para 50\% dos indivíduos expostos ou DL50 hematológica, e a sobrevivência do paciente dependerá, em grande parte, do resultado do enxerto ${ }^{(\mathbf{1 8})}$.

Nos dias de hoje, para a realização da ICT, muitos centros utilizam unidades de ${ }^{60} \mathrm{Co}$ com campos alargados de radiação e grandes distâncias de tratamento entre a fonte e o paciente ${ }^{(\mathbf{3 , 5 , 1 9 , 2 0})}$.

Verificou-se que, a $3 \mathrm{~m}$ de distância, ocorre uniformidade na intensidade da dose em torno de mais ou menos $5 \%^{(\mathbf{3 , 2 0})}$. A ICT realizada em unidades de ${ }^{60} \mathrm{Co}$, numa distância de $3 \mathrm{~m}$, permite que a dose seja máxima à profundidade de $2 \mathrm{~mm}$ (região de "build up"), isto é, na região em que ocorre máxima interação da radiação ionizante com o meio, e de $90 \%$ da dose medida na profundidade de $0,8 \mathrm{~mm}^{(\mathbf{5 , 2 1 , 2 2})}$. Outros estudos relatam como região de "build up" a profundidade de 1,5 mm, também à distância de $3 \mathrm{~m}^{(3)}$. Nas distâncias usuais de tratamento em unidade de ${ }^{60} \mathrm{Co}$ com energia média de 1,25 MeV, o "build up" ocorre em $5 \mathrm{~mm}$ de profundidade ${ }^{\text {(22) }}$.

A homogeneidade da dose é essencial para a técnica de $\mathrm{ICT}^{(3)}$, e variações das doses absorvidas não devem superar $10 \%$, sob o risco de diminuir a eficácia do tratamento $^{(23)}$. Esta determinação das doses durante o ICT tem sido verificada por meio de dosímetros termoluminescentes (TLD), com erro máximo de $\pm 5 \%$ da dose efeti$\mathrm{va}^{(\mathbf{3 , 2 0})}$. Embora uma relação entre a termoluminescência e a exposição aos RX ou raios gama tenha sido observada no início do século passado, a aplicação da termoluminescência na dosimetria das radiações só muito depois foi sugerida, em $1960^{(24)}$.

Randall e Wilkins ${ }^{(\mathbf{2 5})}$ foram os primeiros a desenvolver a teoria matemática para explicar o escape das cargas armadilhadas, tomando como base a distribuição de Maxwell para energias térmicas absorvidas. Essa distribuição representava apenas um 
único pico de emissão de luz. A partir de 1960, o uso da dosimetria termoluminescente ampliou-se rapidamente, devido às vantagens da técnica e à disponibilidade em escala comercial de tipos variados de fósforos (substância luminescente) e de instrumentação ${ }^{(24)}$.

O sulfato de cálcio com disprósio $\left(\mathrm{CaSO}_{4}\right.$ :Dy) é um cristal que apresenta termoluminescência após irradiação, sendo utilizado como dosímetro. Este material é adequado para detectar pequenas doses de radiação e possui alta sensibilidade. Entre os TLD mais utilizados está o fluoreto de lítio com magnésio ou titânio (LiF:Mg,Ti), cuja sensibilidade, quando comparada ao $\mathrm{CaSO}_{4}$ :Dy para radiação gama, é de 30 a 40 vezes inferior. $\mathrm{O}$ sulfato de cálcio nominalmente puro já apresenta emissão termoluminescente, devido a impurezas residuais presentes no cristal, e nessa condição sua resposta é aproximadamente 100 vezes menor que a obtida com o cristal impurificado com disprósio, após ser submetido à mesma dose de radiação ${ }^{(\mathbf{2 6 , 2 7})}$.

No Brasil, o Instituto de Pesquisas Energéticas e Nucleares/Comissão Nacional de Energia Nuclear/São Paulo (Ipen/CNEN/ $\mathrm{SP})$ produz $\mathrm{CaSO}_{4}$ :Dy (0,1\% mol de Dy) pelo método de evaporação, em um sistema selado ${ }^{(28)}$. Esses cristais são triturados, compactados com teflon (para obtenção de resistência mecânica adequada) e sintetizados convenientemente, até se tornarem pastilhas dosimétricas de $0,8 \mathrm{~mm}$ de espessura e com 6 mm de diâmetro ${ }^{(26,29,30)}$.

Dessa forma, o objetivo do presente trabalho é determinar as doses de radiação durante a técnica ICT para condicionamento do TMO, avaliar as variações que podem ocorrer devido às diferenças anatômicas, entre as doses teóricas, calculadas e medidas, utilizando-se TLD, e estabelecer correlação entre a região anatômica irradiada (dose medida) e a dose calculada, com a finalidade de verificar se a dose absorvida na região medial prescrita apresenta tolerância inferior $\mathrm{a} \pm 10 \%$.

\section{MATERIAIS E MÉTODOS}

No presente trabalho foram irradiados nove pacientes de ambos os sexos, portadores de leucemia, para condicionamento de TMO. Considerou-se o tipo de leuce- mia e a fase da doença, se em segunda remissão ou, em alguns casos, recaída. Procedeu-se à mesma metodologia de irradiação em todos os casos (Figura 1).

Para a dosimetria foram utilizados 200 TLD de $\mathrm{CaSO}_{4}: \mathrm{Dy}+$ teflon, fabricados pelo Ipen/CNEN/SP. As leituras foram realizadas utilizando-se leitora Harshaw, modelo 4000A, do Laboratório de Dosimetria do Departamento de Diagnóstico por Imagem da Universidade Federal de São Paulo (LD/DDI-Unifesp).

Os dosímetros foram individualmente calibrados, e para isto foram inicialmente identificados por números e submetidos a uma pré-dose de radiação de intensidade variável, antes do tratamento térmico necessário para preservação de sua estabilidade, reprodutibilidade, sensibilidade, reutilização e eliminação de resíduos provenientes de irradiações anteriores.

Todas as pastilhas foram submetidas à temperatura de $300 \pm 15^{\circ} \mathrm{C}$ por três horas em forno mufla.

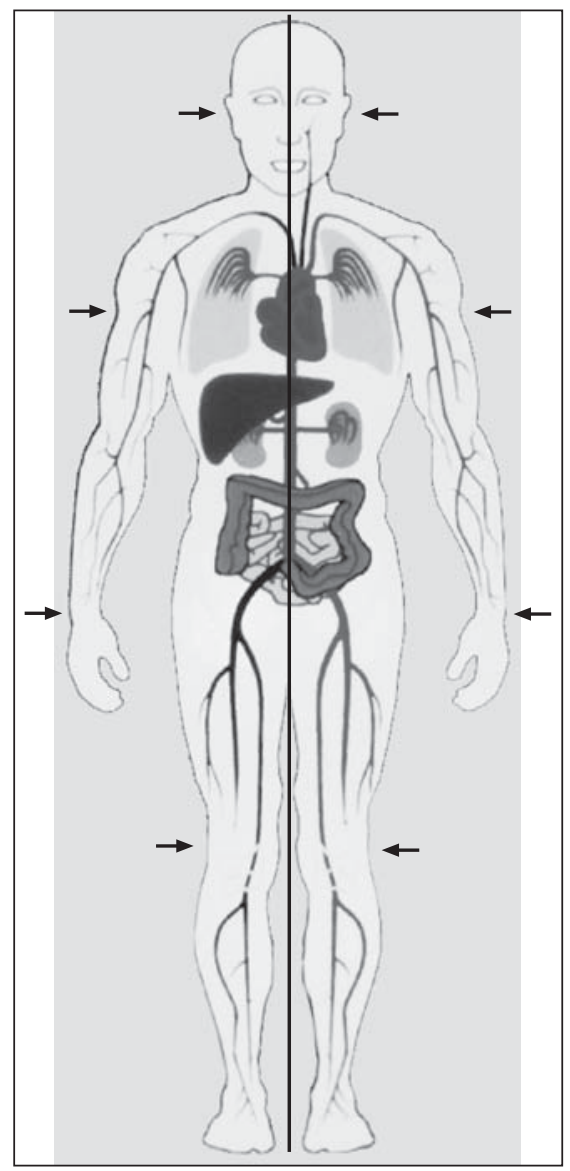

Figura 1. Representação esquemática dos diversos órgãos e a radiação incidente.
$\mathrm{O}$ equipamento utilizado para irradiação das pastilhas TLD e dos pacientes foi uma unidade de ${ }^{60} \mathrm{Co}$ da marca CGR, modelo Alcion II, instalada no Setor de Radioterapia da Unifesp, com taxa de dose média de $0,80 \mathrm{~Gy} / \mathrm{min}$ a $80,5 \mathrm{~cm}$, no campo $10 \times 10 \mathrm{~cm}$. Energia média $(\mathrm{E})$ da radiação g do ${ }^{60} \mathrm{Co:} 1,25 \mathrm{MeV}$.

As pastilhas TLD foram irradiadas no centro do campo $10 \times 10 \mathrm{~cm}$ e identificadas como 1 a 200, com grafite bem fino. Foram separadas aleatoriamente em grupos de nove e seladas com plástico fino. Procedeu-se à irradiação das pastilhas, inicialmente no ar, com a dose de 2 Gy, e posteriormente na água, com a finalidade de conhecer sua resposta no meio espalhador. A irradiação foi realizada a $3 \mathrm{~m}$ de distância, considerando a variação do "build-up" nessa distância. Tanto na irradiação no ar quanto no simulador de água usou-se um superficializador de dose ou fator de crescimento "build-up" (2 $\mathrm{mm})$, para que a dose fosse máxima no cristal. $\mathrm{O}$ processo de preparo e de irradiação das pastilhas foi repetido, por três vezes, para caracterizálas quanto à reprodutibilidade, precisão de resposta e sensibilidade.

Determinou-se a média dos valores individuais das pastilhas e, em função da dose conhecida, calculou-se o fator de conversão ou fator individual da pastilha, isto é, a sensibilidade individual de cada uma por Gy de radiação para a energia do ${ }^{60}$ Co. Os mesmos procedimentos foram repetidos para as pastilhas irradiadas com dose de 2 Gy, no meio espalhador.

Para a verificação da sensibilidade de cada pastilha, levando em conta o espalhamento, estas foram colocadas sobre um simulador de água (tanque de acrílico) de $30 \times 30 \times 40 \mathrm{~cm}$. Determinou-se, assim, um fator, devido ao espalhamento para a energia do ${ }^{60} \mathrm{Co}$.

A irradiação dos pacientes selecionados pela equipe de TMO para condicionamento foi realizada após concordância plena por parte dos pacientes. Procedeu-se à irradiação em corpo inteiro, na posição láterolateral (Figura 2), com o aparelho posicionado de forma a manter a direção da irradiação perpendicular ao paciente. As doses variaram de 2 Gy (uma vez ao dia, dose total de 12 Gy), 2 Gy (duas vezes ao dia, dose total de 12 Gy) e 1,2 Gy (três vezes 


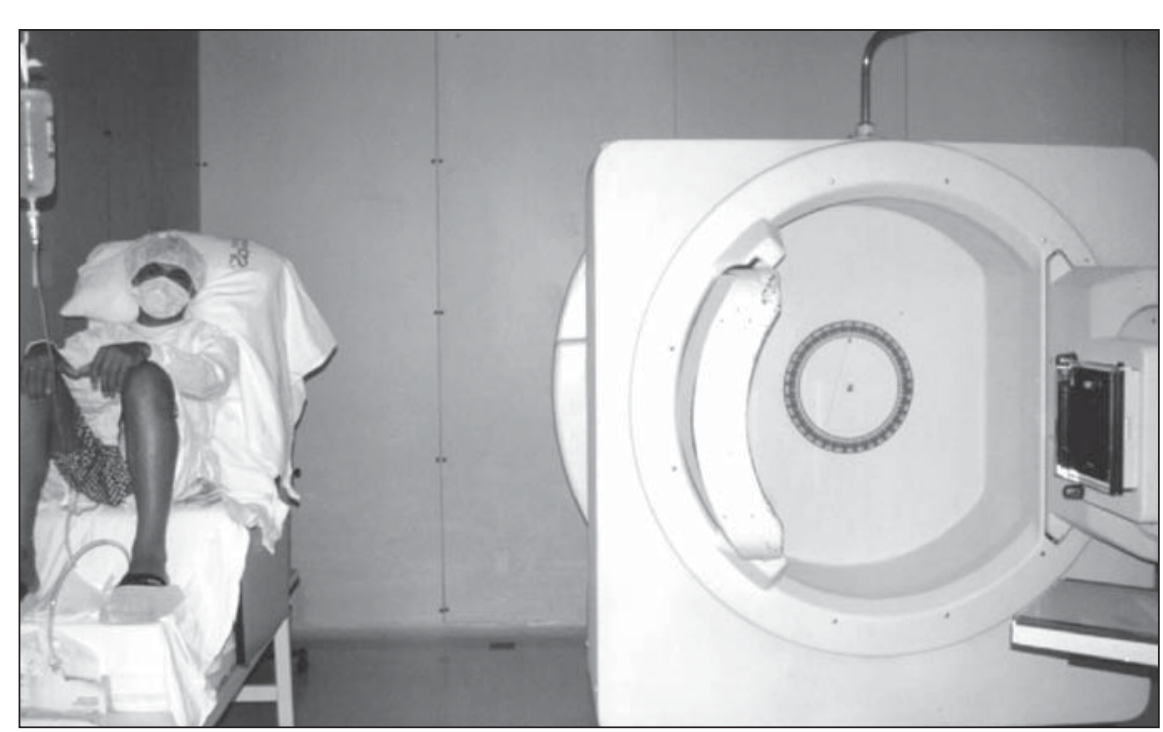

Figura 2. Paciente na posição látero-lateral e aparelho posicionado para manter irradiação perpendicular.

ao dia, dose total de $13,2 \mathrm{~Gy}$ ), sempre com a taxa de dose calculada variando no máximo de 0,03 a $0,08 \mathrm{~Gy} / \mathrm{min}$.

Os valores dos parâmetros utilizados no cálculo da dose na linha média dos pacientes foram registrados em uma ficha modelo. Como nesse tipo de tratamento desejamos irradiar o corpo todo, metade da dose é prescrita para cada lado (direito e esquerdo) e o paciente é irradiado em duas etapas seguidas.

Os dosímetros foram agrupados, em número de cinco, e colocados aleatoriamente na região da cabeça, dos ombros, do tórax, do abdome, da pelve, da coxa, da vulva ou dos testículos e das pernas dos pacientes (Figura 3).

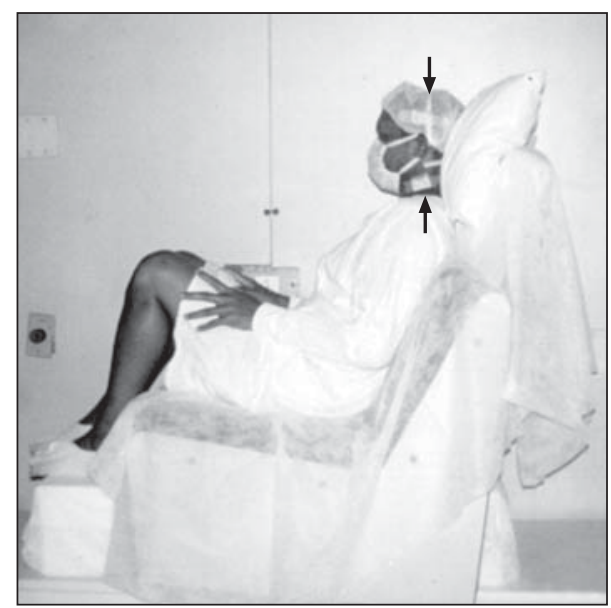

A

Figura 3. A: Dosímetro termoluminescente, paciente posicionado. Verifica-se a existência de TLD na região do pescoço e da cabeça (setas). B: Detalhe dos dosímetros (setas). ção na distância $\mathrm{F}(\mathrm{cm} \times \mathrm{cm}) ;(\mathrm{DLL})_{\mathrm{a}}=$ diâmetro látero-lateral no abdome $(\mathrm{cm})$; $\mathrm{dm}=$ profundidade de equilíbrio eletrônico $\left(0,2 \mathrm{~cm}\right.$ para raios- $\gamma$ do ${ }^{60} \mathrm{Co}$ à distância de $300 \mathrm{~cm}) ; \operatorname{FSP}\left(\mathrm{S}_{\mathrm{t}}\right)=$ fator espalhamento-pico no tórax e abdome.

b) taxa de dose na saída da pele:

$$
\begin{gathered}
D_{a r}(S, F) \times\left\{F /\left[F+\left[(D L L)_{a} / 2\right]-d m\right]\right\}^{2} \\
\times\left\{R T A_{a}\left[(D L L)_{a}-d m, S_{t}\right]\right\}
\end{gathered}
$$

onde: $\mathrm{D}_{\mathrm{ar}}(\mathrm{S}, \mathrm{F})=$ taxa de dose no ar: campo $\mathrm{S}$, distância $\mathrm{F}$, corrigida pela variação fora do eixo central (Gy/min); F = distância fonte-plano sagital médio do paciente $(\mathrm{cm}) ; \mathrm{S}$ = dimensões do campo de irradiação na distância $\mathrm{F}(\mathrm{cm} \times \mathrm{cm}) ;(\mathrm{DLL})_{\mathrm{a}}=$ diâmetro látero-lateral no abdome $(\mathrm{cm})$; $\mathrm{dm}=$ profundidade de equilíbrio eletrônico $(0,2 \mathrm{~cm}$ para raios- $\gamma$ do ${ }^{60} \mathrm{Co}$ à distância de $300 \mathrm{~cm}$ ); $\mathrm{RTA}_{\mathrm{a}}=$ relação tecido-ar na região do abdome; $\mathrm{S}_{\mathrm{t}}=$ campo quadrado equivalente no tórax e abdome $(\mathrm{cm} \times \mathrm{cm})$.

c) taxa de dose no plano sagital médio:

Para calcularmos a taxa de dose no plano sagital médio utilizamos a equação:

$$
D=D_{a r}(S, F) \cdot R T A_{a}\left(\left(D L L_{a}\right) / 2, S_{t}\right)
$$
relação da dose tecido-ar (RTA), definida pela relação entre as doses na água e no ar.

a) taxa de dose na entrada da pele:

$$
\begin{gathered}
D_{a r}(S, F) \times\left\{F /\left[F-\left[(D L L)_{a} / 2\right]+d m\right]\right\}^{2} \\
\times F S P\left(S_{t}\right)
\end{gathered}
$$

onde: $\mathrm{D}_{\mathrm{ar}}(\mathrm{S}, \mathrm{F})=$ taxa de dose no ar: campo $\mathrm{S}$, distância $\mathrm{F}$, corrigida pela variação fora do eixo cental (Gy/min); F = distância fonte-plano sagital médio do paciente (cm); $\mathrm{S}=$ dimensões do campo de irradia-

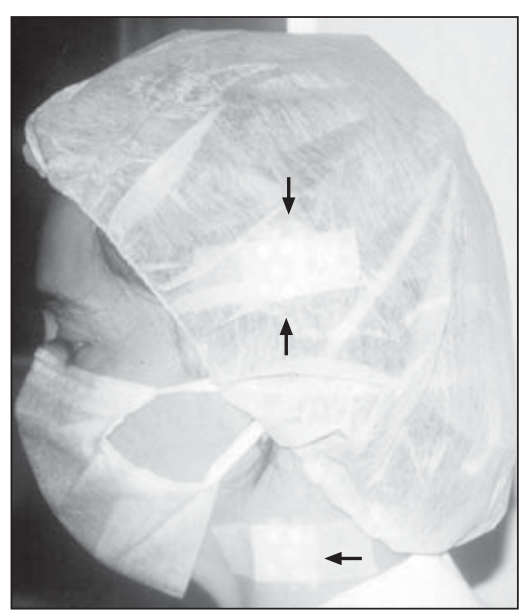
B

d) total de dose administrada:

A dose total administrada na profundidade foi calculada pela soma das taxas de dose obtidas nas equações 1 e 2 , multiplicada pelo produto do tempo total de aplicação e o número de aplicações realizadas.

No plano sagital, a dose total foi calculada pela soma das taxas de dose incidentes pelos dois lados do paciente (equação 3), multiplicada pelo produto do tempo total aplicação e o número de aplicações realizadas.

Foram repetidas, com a mesma equação utilizada para o abdome, o cálculo da dose na cabeça e no tórax, observando sempre o espalhamento e as distâncias corrigidas para as diferentes dimensões anatômicas do paciente.

Para a análise estatística utilizou-se o teste de Wilcoxon "signed rank sum" (teste de postos com sinais de Wilcoxon).

O teste de postos com sinais de Wilcoxon é aplicado para testar a afirmação de que dois conjuntos de dados dependentes provêm de uma mesma população, e se trata de testes não-paramétricos para dados ordinais, também chamados de livre de distribuição. São usados em situações de 
distribuição assimétrica, independem da distribuição dos dados nas amostras, e usa a mediana como referência. São construídas "caixas", cuja mediana é representada por uma linha no interior delas. Essas "caixas" representam os valores do intervalo interquartil, que se referem aos valores compreendidos entre o percentil 25 e o percentil 75; dessa forma, $50 \%$ dos valores centrais da distribuição encontram-se na média.

\section{RESULTADOS}

Nossos resultados mostram que em $70 \%$ da regiões avaliadas ocorreu variação de dose menor que $5 \%$ (Tabela 1 ) e que em $30 \%$ esta variação foi inferior a $10 \%$ (Tabela 2), quando comparados os valores medidos com aqueles calculados em cada ponto. Na região da cabeça houve absorção, em média, de 14\% da dose administrada, possivelmente em função da calota craniana. Nos pulmões observou-se acrés- cimo de $2 \%$ da dose administrada, levando-se em conta as dimensões dos pulmões já corrigidas para realização dos cálculos.

A distribuição das doses medidas com espalhamento e sem espalhamento, levando-se em conta todas as regiões anatômicas de todos os pacientes, está apresentada no Gráfico 1.

O Gráfico 2 apresenta os resultados das medidas com e sem espalhamento obtidos, comparando os pacientes de porte físico de padrão "normal" com aqueles fora do padrão "normal".

\section{DISCUSSÃO}

A dificuldade da técnica da ICT para TMO, em que são combinados dois tipos de campos de irradiação, ocorre devido à imprecisão de alguns fatores no cálculo da dose absorvida. Entre os últimos, podemos citar a medida da distância em campos laterais, que pode variar de 1 a $2 \mathrm{~cm}$. Doses calculadas para alguns pontos anatômicos

Tabela 1 Variação porcentual das doses medidas e calculadas em relação à desejada, nas diversas regiões do corpo, para os seis pacientes de porte físico de padrão "normal".

\begin{tabular}{|l|c|c|c|}
\hline \multirow{2}{*}{$\begin{array}{c}\text { Regiões } \\
\text { do corpo }\end{array}$} & $\begin{array}{c}\text { Dose calculada } \\
\text { em relação } \\
\text { ao desejado (\%) }\end{array}$ & $\begin{array}{c}\text { Meses medidas } \\
\text { com espalhamento }\end{array}$ & $\begin{array}{c}\text { Doses medidas } \\
\text { sem espalhamento }\end{array}$ \\
\cline { 2 - 4 } Abdome & 7,5 & 13,8 & 6,2 \\
Bacia & 10,8 & 6,6 & $-1,5$ \\
Cabeça & 13,9 & 2,5 & $-2,0$ \\
Coxa & 15,5 & 11,6 & 3,9 \\
Pescoço & 32,8 & 14,7 & 1,5 \\
Testículo & 0 & 7,5 & $-0,8$ \\
Tórax & 15,9 & 13,5 & 6,2 \\
\hline Média & 13,7 & 10 & 1,9 \\
\hline
\end{tabular}

Tabela 2 Variação porcentual das doses medidas e calculadas em relação à desejada, nas diversas regiões do corpo, para os três pacientes de porte físico fora do padrão "normal".

\begin{tabular}{|l|c|c|c|}
\hline \multirow{2}{*}{$\begin{array}{c}\text { Regiões } \\
\text { do corpo }\end{array}$} & $\begin{array}{c}\text { Dose calculada } \\
\text { em relação } \\
\text { ao desejado (\%) }\end{array}$ & $\begin{array}{c}|c| \\
\text { Doses medidas } \\
\text { com espalhamento }\end{array}$ & $\begin{array}{c}\text { Doses medidas } \\
\text { sem espalhamento }\end{array}$ \\
\cline { 2 - 4 } Abdome & 0,0 & 47,5 & 36,2 \\
Bacia & $-2,5$ & 46,0 & 34,8 \\
Cabeça & 10,4 & 39,3 & 28,4 \\
Perna & $-4,3$ & 50,8 & 39,1 \\
Coxa & $-3,3$ & 46,6 & 35,5 \\
Pescoço & 18,7 & 47,0 & 35,8 \\
Testículo & 0,0 & 33,2 & 31,8 \\
Tórax & 1,9 & 40,7 & 32,7 \\
\hline Média & 2,6 & 43,9 & 34,3 \\
\hline
\end{tabular}

podem apresentar uma variação de cerca de $15 \%$, apenas com o erro da reprodução no posicionamento, a cada fração de dose administrada. Esta variação da dose ou imprecisão (soma de erros) pode causar prejuízo no tratamento ${ }^{(\mathbf{2 0})}$.

Os nossos resultados referentes aos seis pacientes considerados dentro do padrão "normal" evidenciaram, em relação aos valores desejados e estabelecidos na terapêutica, variações de doses de $1,93 \%$ sem espalhamento e 10,07\% com espalhamento. Em relação ao valor calculado, a variação foi de 13,79\%. Em média, a dose absorvida apresentou variabilidade de aproximadamente $6 \%$. Este resultado está abaixo do esperado, de acordo com a literatura, que aceita uma não homogeneidade de dose de $10 \%^{(\mathbf{5})}$. Quanto aos três pacientes considerados de padrão fora do "normal", observamos variação máxima de dose de $39,11 \%$ a 3 m de distância, calculada na região sagital do paciente, com e sem espalhamento, na entrada e na saída do paciente. Estes dados confirmam a necessidade de confecção de filtros compensadores de dose, próprios para cada região. Conforme indicado na literatura, variações acima de $20 \%$ ocorrem quanto maior for o diâmetro látero-lateral ${ }^{(\mathbf{5 , 2 3})}$.

A relevante preocupação quanto à dose verdadeira ocorre devido ao espalhamento produzido pelo feixe de radiação na entrada e na saída do paciente ${ }^{(5)}$. Deseja-se minimizar o erro de medida em função da região de máxima interação da radiação com os tecidos da região de "build up", a fim de evitar variações de dose na pele do paciente. Existem, na literatura, diferentes técnicas para realização dessas avaliações de dose absorvida ${ }^{(\mathbf{2 0})}$.

No presente trabalho utilizamos os TLD, pois estes apresentam maior eficiência na medição de dose quando comparados com os outros sistemas, possuem menor influência no que se refere a temperatura, taxa dose, alta energia, e constituem instrumentos de medição adequados para dosimetria in vivo ${ }^{(5)}$. A dose na entrada $\mathrm{e}$ saída do feixe foi medida e a porcentagem de erro foi determinada com vários sistemas. Os dosímetros foram posicionados na superfície das diferentes regiões anatômicas dos pacientes. Verificamos a entrada e a saída da dose em cada região e não nos 


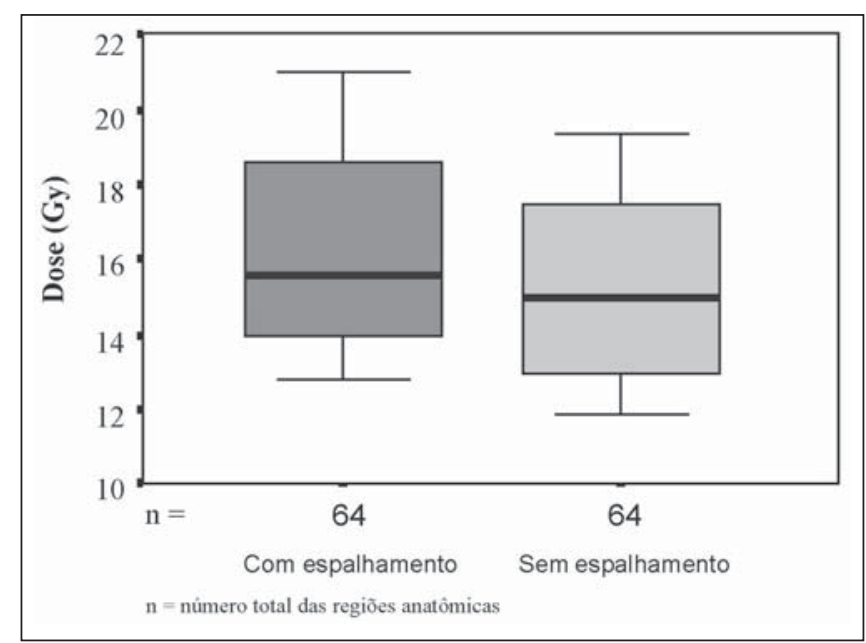

Gráfico 1. Representação gráfica da variação das doses medidas em Gy, em todos os pacientes, para todas as regiões anatômicas, com e sem espalhamento.

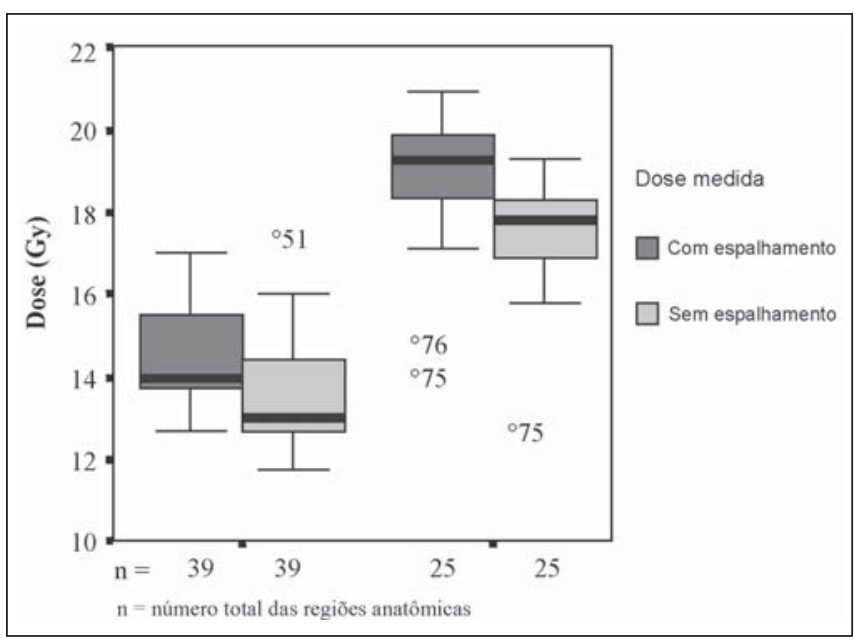

Gráfico 2. Representação gráfica da variação das doses medidas em Gy, comparando todos os pacientes de porte físico de padrão "normal" e aqueles fora do padrão, com e sem espalhamento. preocupamos com a colocação dos dosímetros em profundidade. Esse procedimento foi baseado na literatura, que refere que para um campo de $150 \times 150 \mathrm{~cm}$, à distância de aproximadamente $300 \mathrm{~cm}$, a porcentagem de dose máxima para o ${ }^{60} \mathrm{Co}$ ocorre a $2 \mathrm{~mm}$, e a porcentagem de dose que varia da máxima a $90 \%$ ocorre a $0,8 \mathrm{~mm}$. A literatura informa também que para o ${ }^{60} \mathrm{Co}$, em campo paralelo oposto com profundidade maior de $25 \mathrm{~cm}, 90 \%$ da dose ocorre a $1 \mathrm{~mm}$ e a dose "build up" é considerada como dose absorvida na pele. Em nosso experimento, o cristal $\left(\mathrm{CaSO}_{4}\right)+$ plástico protetor e fita de micropore resultam numa espessura de $2 \mathrm{~mm}$. Ela é suficiente para a obtenção de medições de dose nos locais onde foram posicionados os dosímetros e nas condições do aparelho utilizado.

De acordo com a Physics Conference $(1988)^{(5)}$, para espessuras ou diâmetros látero-laterais acima de $28 \mathrm{~cm}$, doses absorvidas com variações maiores que $24 \%$ podem ocorrer nos pulmões. De acordo com nossos dados, encontramos nos pulmões variação máxima de dose medida, em relação à desejada, de 13,54\%. Estes resultados estão, portanto, dentro dos valores aceitos. A calibração adequada dos TLD é de grande relevância e optamos por usar calibração individual das pastilhas para as avaliações de dose absorvida, minimizando, dessa forma, os erros intrínsecos à calibração em grupo. Para dosimetria in vivo, os TLD, colocados na pele dos pacientes, são adequados para a verificação da dose mediana e mostram erro menor que $3 \%$ quando os resultados são comparados com métodos de equação e de medida ${ }^{(20)}$.

Os dados obtidos neste trabalho reforçam a idéia de que o uso da dosimetria termoluminescente para a dosimetria in vivo constitui um método preciso de verificação de dose absorvida e deve ser usada para todos os casos de ICT em confronto com os cálculos teóricos. Devido à estrutura física externa e interna dos pacientes variar amiúde, grandes variações de dose podem ocorrer, o que justifica a confirmação das doses por este método. Na clínica, esta técnica deve ser realizada, no mínimo, em $50 \%$ das vezes em que o paciente for submetido à ICT. Havendo condições e colaboração por parte dos pacientes, os dosímetros devem ser também colocados em cavidades internas $^{(7)}$.

\section{CONCLUSÕES}

Com base nos resultados obtidos, acreditamos ser lícito que:

1. Para pacientes com diâmetro láterolateral acima de $34 \mathrm{~cm}$, principalmente em tórax, abdome e bacia, é recomendado o uso de filtros compensadores, com a finalidade de diminuir as doses e obter valores aceitáveis de distribuição dessas doses.

2. Os TLD devem ser calibrados com espalhamento, ou seja, deve-se criar uma superfície simuladora do meio biológico. Nossos resultados mostram que a calibração com espalhamento, em relação à cali- bração tradicional no ar, aproximou-se mais dos valores reais.

3. Em vista do exposto, existe confiabilidade no tratamento realizado para qualquer seguimento anatômico, e a dose total desejada, durante a ICT, foi obtida e apresentou variação menor que $\pm 10 \%$.

\section{REFERÊNCIAS}

1. Segreto RA, Segreto HRC. Radiação ionizante e resposta celular: atualidades. Radiol Bras 1997;30: 301-7.

2. Salvajoli JV. Radioterapia em oncologia. São Paulo, SP: Editora Médica e Científica, 1999:118193.

3. Glasgow GP. The dosimetry of fixed, single source hemibody and total body irradiators. Med Phys 1982;9:311-23.

4. Rider WD, Van Dyk J. Total and partial body irradiation. In: Blechen NM, Glatsein E, Haybittle JL, eds. Radiotherapy treatment planning. New York, NY: Marcel Dekker, 1983:559-94.

5. Zuzag T. Physics conference: total and hemibody photon therapy. Review of treatment techniques in Europe. Radiother Oncol 1988;9:91-106.

6. Thomas ED, Clift RA, Hersman J, et al. Marrow transplantation for acute nonlymphoblastic leukemia in first remission using fractionated or singledose irradiation. Int J Radiat Oncol Biol Phys1982; $8: 817-21$.

7. Lawton CA. Radiation therapy for bone marrow transplantation. In: Cox JD, ed. Moss' radiation oncology: rationale, technique, results. 7th ed. St. Louis, MO: Mosby-Year Book, 1994:937-50.

8. Shank BM. Total body irradiation for bone marrow transplantation. 35th Annual Scientific Meeting of the American Society for Therapeutic Radiology and Oncology, 1993.

9. Singer CRJ, Goldstone AH. Clinic studies of ABMT in non-Hodgkin's lymphoma. Clin Haematol $1986 ; 15: 105-50$.

10. Oliveira HP. Transplante de medula óssea. Rio de Janeiro, RJ: Editora Atheneu, 885:561-77.

11. Clift RA, Buckner CD, Appelbaum FR. Allogeneic marrow transplantation in patients with acute 
myeloid leukemia in first remission: a randomized trial of two irradiation regimens. Blood 1990;76: 1867-71.

12. Ganem G, Kuentz M, Bernaudin F. Central nervous system relapses after bone marrow transplantation for acute lymphoblastic leukemia in remission. Cancer 1989;64:1796-804.

13. Jillella AP, Doria R, Khan K, et al. Cyclophosphamide, cytosine arabinoside and TBI as a conditioning regimen for allogeneic bone marrow transplantation in patients with leukemia. Bone Marrow Transplant 1999;23:1095-100.

14. Gerrard GE, Vail A, Taylor RE, et al. Toxicity and dosimetry of fractionated total body irradiation prior to allogeneic bone marrow transplantation using a straightforward radiotherapy technique. Clin Oncol 1998;10:379-83.

15. Thomas ED, Storb R, Clift RA, et al. Bone marrow transplantation (second of two parts). N Engl J Med 1975;292:895-902.

16. Shank B, Hopfan S, Kim JH, et al. Hyperfractionated total body irradiation for bone marrow transplantation. I. Early results in leukemia patients. Int J Radiat Oncol Biol Phys 1981;7:1109-15.

17. Trigg ME, Billing R, Sondel PM, et al. Clinical trial depleting $\mathrm{T}$ lymphocytes from donor marrow for matched and mismatched allogeneic bone marrow transplant. Cancer Treat Rep 1985;69:377-86.

18. Tubiana M, Frindel E, Croizat H, Parmentier C. Effects of radiations on bone marrow. Pathol Biol 1979;27:326-34.

19. Dominique C, Schwartz LH, Lescrainier J, et al. A modified ${ }^{60}$ Co teletherapy: unit for total body irradiation. Int J Radiat Oncol Biol Phys 1995;33:9517.

20. Malicki J. The accuracy of dose determination during total body irradiation. Strahlenther Onkol 1999; 175:208-12.

21. Pereira SG, Ceccati ER, Almeida CE. Avaliação da dose-superfície e região de "build up" para raios gama de cobalto-60. Radiol Bras 1981;14:41-4.

22. McCullough EC. A measurement and analysis of build up region dose for open field photon beams (cobalt-60 through 24 MV). Med Dosim 1994;19: $5-14$.

23. Van Dyk J. Whole and partial body radiotherapy: physical considerations. In: Wright A, Boyer AL, eds. Advances irradiation therapy treatment planning. Med Phys Monogr Ser 9, 1983:403-26.

24. Da Rosa LAR. Dosimetria termoluminescente: teoria, materiais e aplicações. (Monografia). São Paulo, SP, 1978
25. Randall JT, Wilkins MHF. Phosphorescence and electron traps. I. The study of trap distributions. Proc Roy Soc (London) 1945;A184:366-9.

26. Daros KAC. Estudo da resposta TL de pastilhas de $\mathrm{CaSO}_{4}$ :Dy com grafite para detecção de radiação beta e fótons de energias baixas. (Tese de Mestrado). São Paulo, SP: Universidade Federal de São Paulo, 1997

27. Grigoletto T, Lordello AR. Determinação espectrográfica de disprósio, dopante de sulfato de cálcio utilizado como material dosimétrico. São Paulo, SP: Instituto de Pesquisas Energéticas e Nucleares, 1984.

28. Campos LL. V Curso para o credenciamento de pessoas físicas para supervisionar aplicação das medidas e regulamentos apropriados de proteção radiológica na indústria: dosimetria termolumines cente. São Paulo, SP: Instituto de Pesquisas Energéticas e Nucleares, 1980

29. Da Rosa LAR, Caldas LVE, Cunha PG. Thermoluminescence characteristics of ultra-thin $\mathrm{CaSO}_{4}$ :Dy beta radiation detection. Radiat Prot Dosimetry 1986;14:329-31.

30. Campos LL, Lima MF. Dosimetric properties of $\mathrm{CaSO}_{4}:$ Dy teflon pellets produced at IPEN. Radiat Prot Dosimetry 1986;14:333-5. 